\title{
Using noncircular gears for the unloading door kinematics modification
}

\author{
Mircea Niculescu*, and Laurenția Andrei \\ "Dunărea de Jos" University of Galaţi, Faculty of Engineering, Department of Mechanical \\ Engineering, str. Domnească nr. 111, 800201, Romania
}

\begin{abstract}
The paper introduces a new kinematics of the billet reheating furnace unloading door, by using a noncircular gear train in the drive mechanism. The main objective of the kinematics modification is to reduce the heat loss by increasing the unloading door speed, during the stroke, while kept it at the conventional value at the beginning and end of stroke. In order to modify the door kinematics, the drive mechanism output motion is controlled by noncircular gears whose transmission ratio is defined as a hybrid multiparameters function. A study is developed to analyse the influence of the defining parameters on the variation of the gears transmission ratio and on the discharging door speed, respectively. Based on the analytical study, a certain law of variation was chosen to describe the mechanism kinematics; in accordance to the kinematics variation, the mating noncircular centrodes/pitch curves were modelled and the gears teeth are generated, considering constant circular pitch and constant pressure angle, respectively.
\end{abstract}

\section{Introduction}

Due to nowadays ease of design and manufacture, compactness and low costs, noncircular gears have become an efficient solution for the driving mechanisms of closing/regulation industrial applications, where velocities are subjected to variations or motions of driven elements should obey imposed trajectories [1]. For example, Y. Takahiro proposes a vehicle window regulator that varies the window speed, increasing it as the window gets near to its fully closed position [2]. The regulator is based on the meshing of two noncircular gears of circular eccentric, elliptical and logarithmically helical shapes. J. P Nolta proposes a noncircular gears pair defined by a transmission ratio that varies from a linear function to a non-linear one, proportional to an exponential function, in order to influence the closing element of the motor vehicle climate control system [3]. G. Vonhausen proposes noncircular gears as driving mechanism for an actuation valve, in order to adjust the liquid flow [4], while H.C Buchanan uses noncircular gears to adjust the windshield wiper speed [5].

The authors of this paper are taking into account the usage of noncircular gears for the

*Corresponding author: mircea.niculescu@yahoo.com 
furnace discharging door speed with the aim of reducing the heat loss during the specific stroke.

\section{Modified kinematics of the unloading door drive mechanism}

In hot-rolling process, the flow first operation is the billets heating, at $1250^{\circ} \mathrm{C}$ temperature, into specialised furnace. The furnace is successively fed with billets, one by one, by means of Push Machine. The billets are discharged through the front side of the furnace, at a rate of one piece at every 2-3 minutes; every time, the discharge door is opened, in order to enable the fall of billets on the roller table, and then is closed. Taking into account that the billets discharging period is shorter (1-2 seconds) than the time required for the door opening and closing process (12 seconds), a large amount of heat is lost through the open door space. In order to reduce this energy loss, the door kinematics could be varied by introducing a noncircular gears train within the door driving mechanism; the authors intend to keep at constant designed value the door velocity while opening and closing the door and to increase the door velocity during the stroke.

\subsection{The analysis of the actual door mechanism kinematics}

The discharge door of the billets heating furnace is driven by an asynchronous motor (1) and a mechanism consisting of a worm gear (2) and a driving chain (4), as shown in Figure 1 [6]. The door (8) is lifted by means of chains (7) that are wound on the wheels (6). The total transmission ratio is 140 assures a door lifting velocity of $0.08 \mathrm{~m} / \mathrm{s}$. This velocity is required when the furnace door is opened and closed, in order to avoid the mechanical impacts on the furnace frontal structure. To open the door, the motor shaft is clockwise rotated; to close the door, the motor shaft rotational motion is reversed. The door stroke is electrically limited by a controller.

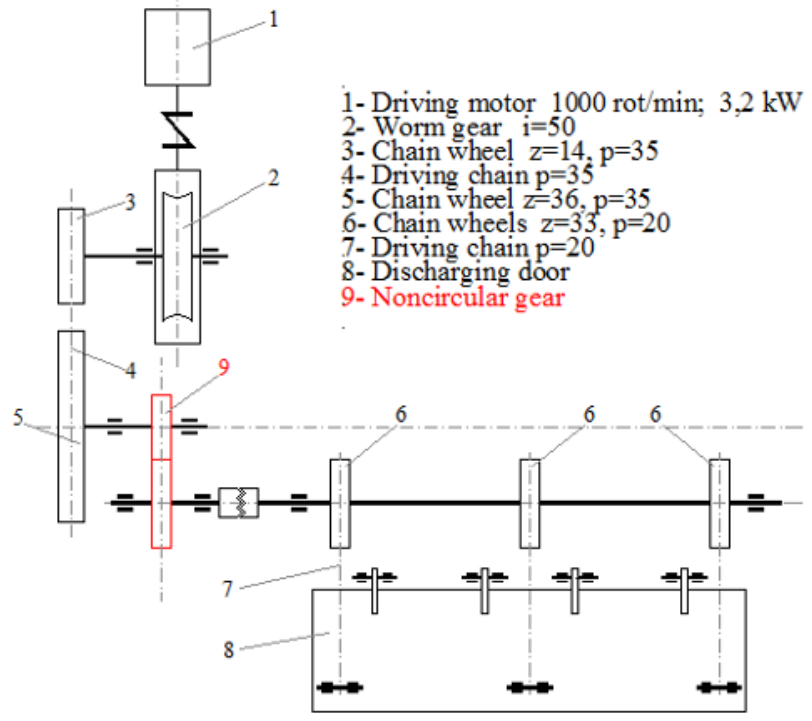

Fig. 1. The modified driving mechanisms of the discharging door, by inserting noncircular gears.

\subsection{The modified kinematics of the door driving mechanism}


In order to increase the door angular velocity, a noncircular gears train is placed within the door driving mechanisms, between the driving chain (4) and the driving shaft of the wheels (6), as shown in Figure 1. The noncircular gears (9) perform less than one complete rotation as long as the total rotational angle of the driven gear should be $\theta_{2 \mathrm{t}}=308.3^{\circ}$ (corresponding to the opening door angle of $45^{\circ}$ ). The gears geometry consists of a combination of circular and noncircular areas, in correspondence to the desirable transmission ratio variation.

In order to achieve smooth opening and closing motion for the discharging door, the transmission ratio variation is divided into three phases: constant conventional value of 1:1 at the beginning of stroke, nonlinear increase to a maximum value during the opening process and constant value again, at the high rate of $2: 1$, till the discharging door is completely opened. Reversing de motor shaft rotational motion, the door is closed by a high constant speed for a while, and then the speed is gradually reduced to the conventional value and kept at this value while the door is clos

\subsection{The influence of noncircular gears defining parameters, on the door mechanism kinematics}

In order to modify the kinematics of the door driving mechanism, the noncircular gear transmission ratio is defined as a multiparameters hybrid function:

$$
i_{21}\left(\varphi_{1}\right)=\left\{\begin{array}{cc}
1, & \varphi_{1} \in\left[0, \varphi_{u}\right] \\
\frac{i_{\text {max }}+1}{2}+\frac{1-i_{\text {max }}}{2} \cdot \sin \left(\frac{\pi}{\varphi_{v}-\varphi_{u}} \cdot \varphi_{1}+\frac{\pi\left(\varphi_{v}-\varphi_{u}\right)}{2\left(\varphi_{v}-\varphi_{u}\right)}\right), & \varphi_{1} \in\left[\varphi_{u}, \varphi_{v}\right] \\
i_{\text {max }}, & \varphi_{1} \in\left[\varphi_{v}, \varphi_{1 \mathrm{t}}\right]
\end{array}\right.
$$

where $i_{\max }$ is the maximum transmission ratio achieved during the door stroke; $\varphi_{u}-$ the driving gear rotational angle while the motion is transmitted by the constant conventional ratio $(1: 1) ; \varphi_{v}$ - the driving gear rotational angle where the variable motion turns into an uniform one; $\varphi_{1 t}$ - the driving gear rotational angle when the door is closed, corresponding to the defined angle of the driven gear $\varphi_{2 t}=308.3^{\circ}$. The variation of the transmission ratio is chosen so that a continuous, positive and derivative function is defined.

The rotation angle of the driven gear is expressed by [1]:

$$
\varphi_{2}\left(\varphi_{1}\right)=\int_{0}^{\varphi_{1}} i_{21}\left(\varphi_{1}\right) d \varphi
$$

Imposing that $\varphi_{2}>0, \varphi_{2 \mathrm{t}}=308.3^{\circ}$ and considering the variation of the gear transmission ratio from equation (1), the total rotation angle of the driving gear could be calculated by equation (3)

$$
\varphi_{1 t}=\frac{\varphi_{2 t}}{i_{\max }}+\frac{i_{\max }-1}{2 i_{\max }} \cdot\left(\varphi_{u}+\varphi_{v}\right)
$$

Figure 2 illustrates the results of an initial study that is developed to analyse the influence of the $i_{\max }, \varphi_{u}$ and $\varphi_{v}$ defining parameters on the transmission ratio variation. As noticed in Figure 2, the transmission ratio variation, from $i_{\min }=1$ to its maximum value, depends on the angular difference $\varphi_{v}-\varphi_{u}$; increasing the angular difference, as shown in Figures $2 \mathrm{a}, \mathrm{b}$, the transmission ratio variation gets smoother, the slope and the door acceleration being reduced.

Also, the increase of the maximum transmission ratio, as seen in Figure 2c, increases the driving gear total rotation angle; this increase is not an issue for the mechanism 
kinematics, taking into account that the driving gear doesn't perform a full rotation; Choosing a higher ratio, $i_{\max }$, the technological conditions are improved as the time when the door is open and the heat is lost, respectively, are reduced.

Figure 3 illustrates the influence of the transmission ratio defining parameters on the discharging door speed. The parameters $\varphi_{v}$ and $\varphi_{u}$, especially their difference $\varphi_{v}-\varphi_{u}$, have a similar influence on the door velocity, $v_{u}$, as they have on the transmission ratio. The door velocity was calculated based on the angular velocity of the driving motor and considering the total value of the transmission ratio from the driving mechanism shown in Figure 1.

Considering the above analysis, the following initial parameters were chosen: $\varphi_{u}=10^{\circ}$, $\varphi_{v} \in\left[60^{\circ}, 90^{\circ}\right]$ and $i_{\max }=2$.

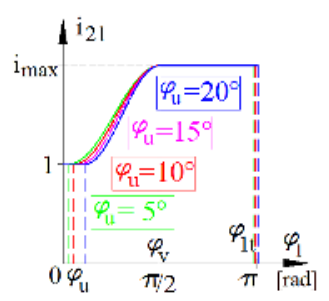

a) $i_{\max }=2 ; \varphi_{u}=5^{\circ}$

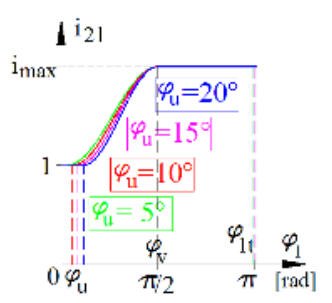

b) $i_{\max }=3 ; \varphi_{v}=90^{\circ}$

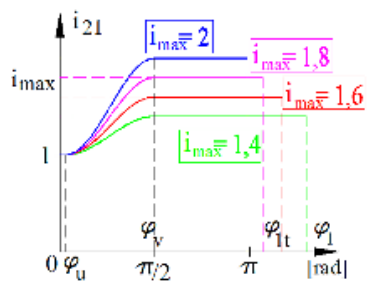

c) $\varphi_{u}=5^{\circ} ; \varphi_{v}=90^{\circ}$

Fig. 2. The influence of the transmission ratio defining parameters $\varphi_{u}(\mathrm{a}), \varphi_{v}(\mathrm{~b})$ and $i_{\max }(\mathrm{c})$ on the transmission ratio variation.

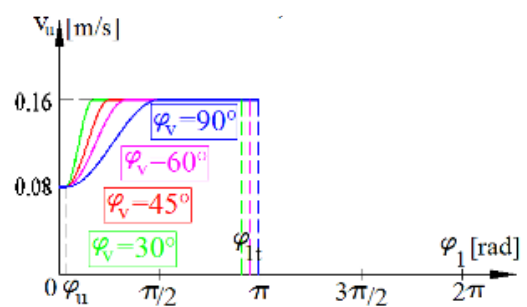

a) $i_{\max }=2 ; \varphi_{u}=5^{\circ}$

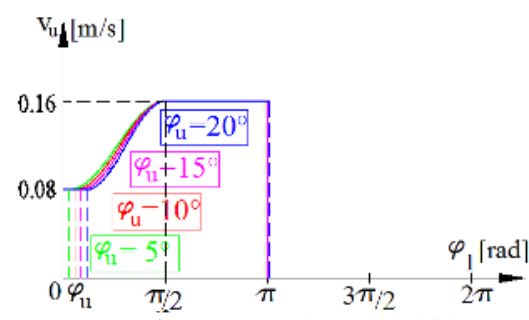

b) $i_{\max }=2 ; \varphi_{v}=90^{\circ}$

Fig. 3. The influence of the transmission ratio defining parameters $\varphi_{u}(\mathrm{a}), \varphi_{v}(\mathrm{~b})$ and $i_{\max }$ (c) on the door lifting velocity.

\section{Non-circular gear generation}

The main steps required within the noncircular gears generation process are (i) the modelling of the mating noncircular centrodes/pitch curves and (ii) the teeth generation.

\subsection{Noncircular pitch curves modelling}

The noncircular gears pitch curves that assure the transmission ratio defined by the equation (1), have the following polar equations [1], [7]:

$$
\begin{gathered}
r_{1}\left(\varphi_{1}\right)=\frac{D}{1+i_{21}\left(\varphi_{1}\right)} \\
r_{2}\left(\varphi_{2}\left(\varphi_{1}\right)\right)=D \frac{i_{21}\left(\varphi_{1}\right)}{1+i_{21}\left(\varphi_{1}\right)}
\end{gathered}
$$


where $\varphi_{1}$ is the driving centrode polar angle, uniformly increased; $\varphi_{2}-$ the driven centrode polar angle, varying in accordance with equation (2); $D$ - the gears center distance.

Based on the equations (4) and (5), two pairs of noncircular pitch curves are modelled for the initial data: the functioning cycle dividing angles $\varphi_{u}=10^{\circ}, \varphi_{v}=60^{\circ}$ and $90^{\circ}$ respectively, the transmission ratio is varied within the limits $i_{\min }=1$ and $i_{\max }=2$, and the gear center distance is $D=250 \mathrm{~mm}$. The pitch curves illustrated in Figure 4 are modelled in AutoCAD environment, using an AutoLISP code.

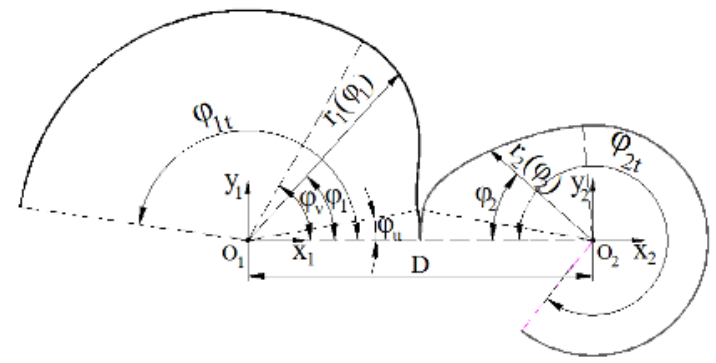

a) $\varphi_{u}=10^{\circ}, \varphi_{v}=90^{\circ}, i_{\max }=2$

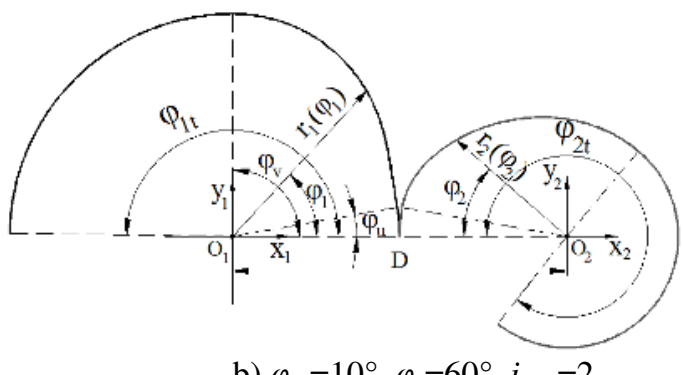

b) $\varphi_{u}=10^{\circ}, \varphi_{v}=60^{\circ}, i_{\max }=2$

Fig. 4. Pitch curves of noncircular gears.

As illustrated, the chosen transmission ratio parameters leads to open noncircular centrodes, with favourable geometry, consisting of circular arcs and convex noncircular arcs, with a reduced area. The driving centrode shape exhibits a concave zone whose curvature has shown to be permissive for the further tooth flanks generation.

\subsection{Gear teeth generation}

For the gear teeth flanks generation, an analytical procedure is applied considering the local geometry of the noncircular pitch curve and the profile of a "single tooth rack cutter" that rolls over the pitch curve. Following the theory presented in [7], the coordinates of the tooth flank points are expressed by equation (6)

$$
\begin{aligned}
& x_{1 i j}=r_{1}\left(\varphi_{1 i j}\right) \cdot \cos \varphi_{1 i j} \pm s_{i j} \cdot \cos \alpha \cdot \cos \left(\mu_{i j}+\alpha+\varphi_{1 i j}\right) \\
& y_{1 i j}=r_{1}\left(\varphi_{1 i j}\right) \cdot \sin \varphi_{1 i j} \pm s_{i j} \cdot \cos \alpha \cdot \sin \left(\mu_{i j}+\alpha+\varphi_{1 i j}\right)
\end{aligned}
$$

where $r_{l i j}, \varphi_{l i j}$ are the polar coordinates of the instantaneous center of rotation; $s_{i j}-$ the rolling distance along the current tangent line; $\mu_{i j}$ - the angle of the current tangent relative to the positioning vector; $\alpha$ - the standard pressure angle $\left(20^{\circ}\right)$; (-) sign is for the gear tooth addendum and $(+)$ sign is for the deddendum segment, when the rolling is counter clockwise performed [7]. Similar equations are written for the driving gear tooth opposite flank points. 
Based on the driving gear tooth flanks profiles, the driven gear tooth conjugate profiles are analytically expressed considering the gears meshing [7]. Using the coordinate transformation, the driven gear conjugate tooth flanks are expressed, in its fixed coordinate system, by equation (7), where $\varphi_{2 i j}$ is the driven gear rotational angle, defined by equation (2).

$$
\left[\begin{array}{l}
x_{2 i j} \\
y_{2 i j}
\end{array}\right]=\left[\begin{array}{cc}
\cos \varphi_{2 i j} & \sin \varphi_{2 i j} \\
-\sin \varphi_{2 i j} & \cos \varphi_{2 i j}
\end{array}\right] \cdot\left(\left[\begin{array}{c}
-D \\
0
\end{array}\right]+\left[\begin{array}{cc}
\cos \varphi_{1 i j} & \sin \varphi_{1 i j} \\
-\sin \varphi_{1 i j} & \cos \varphi_{1 i j}
\end{array}\right] \cdot\left[\begin{array}{l}
x_{1 i j} \\
y_{1 i j}
\end{array}\right]\right)
$$

AutoLISP codes automatically generated the noncircular gears teeth flanks, as illustrated in Figure 5. Constant circular pitch and pressure angle have been considered during the teeth generation. In Figure 6 the noncircular gears sections are completed by additional editing operations, i.e. the teeth addendum and dedendum were considered at $m$ and $1,25 m$, respectively ( $m$ - the gear modulus), and the tooth root is filleted by a $0,25 m$ radius.

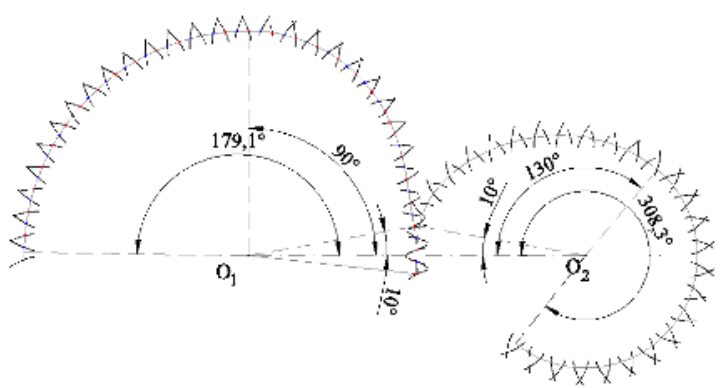

a) $\varphi_{u}=10^{\circ}, \varphi_{v}=90^{\circ}, i_{\max }=2$

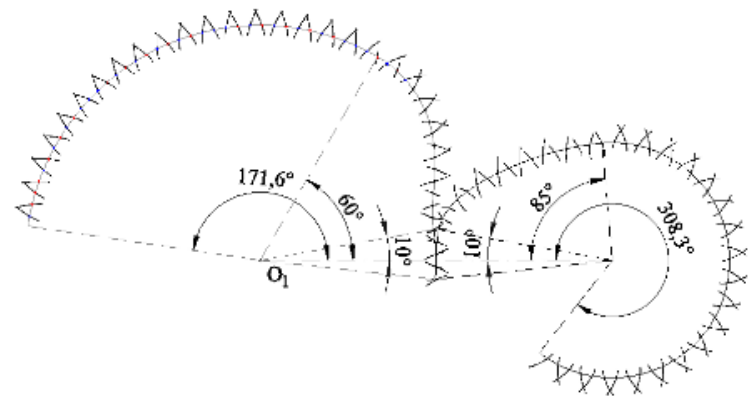

b) $\varphi_{u}=10^{\circ}, \varphi_{v}=60^{\circ}, i_{\max }=2$

Fig. 5. The teeth flanks generation of the noncircular gear.

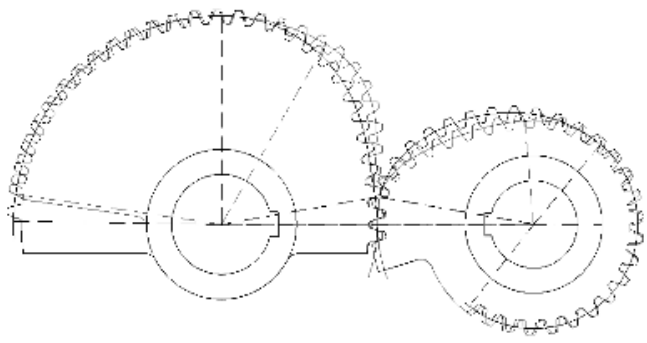

Fig. 6. Comparison of the noncircular gears, in both designed cases. 


\section{Conclusions}

The paper presents a modified kinematics for the unloading door drive mechanism of the billets reheating furnace, in order to reduce the heat loss; the modified kinematics is achieved from a noncircular gear train whose transmission ratio is defined as a hybrid function with multiple parameters; the flexibility of the gears design is therefore increased and enabled to consider the technological requirements. Analysing the influence of the transmission ratio parameters on the door relative velocity, proper values were chosen and introduced into the further noncircular gear design. Original AutoLISP codes were used for the analytical studies and geometric modelling of the noncircular gears.

\section{References}

1. F.L. Litvin, Noncircular Gears Design and Generation, Cambridge University Press (2009)

2. Y. Takahiro, et al., United States Patent 4998379 Mar 12 (1991)

3. J. P. Nolta, United States Patent US 2014/0000397 A1 (2014)

4. D.Vonhausen, United States Patent 4638977 (1987)

5. H.C. Buchanan, et al., United States Patent 4543839 Oct 1 (1985)

6. IPROLAM project. no 505.30-012 (1977)

7. M. Niculescu, L. Andrei, 7th International Conference on Advanced Concepts in Mechanical Engineering, Iasi (2016) 